Preprint typeset in JHEP style - HYPER VERSION

\title{
Brane Interaction as the Origin of Inflation
}

\author{
Nicholas Jones, Horace Stoica and S.-H. Henry Tye \\ Laboratory of Elementary Particle Physics, Cornell University, Ithaca, NY 14853 \\ E-mail: nick.jones@cornell.edu, fhs3@mail.lns .cornell.edu, \\ tye@mail.lns.cornell.edu
}

\begin{abstract}
We reanalyze brane inflation with brane-brane interactions at an angle, which include the special case of brane-anti-brane interaction. If nature is described by a stringy realization of the brane world scenario today (with arbitrary compactification), and if some additional branes were present in the early universe, we find that an inflationary epoch is generically quite natural, ending with a big bang when the last branes collide. In an interesting brane inflationary scenario suggested by generic string model-building, we use the density perturbation observed in the cosmic microwave background and the coupling unification to find that the string scale is comparable to the GUT scale.
\end{abstract}

KeYwords: Inflation, Brane World, String Theory, Cosmology. 


\section{Contents}

1. Introduction 1

2. Effective Action for Brane-Stacks 5

3. Braneworld at the Start of Inflation

3.1 Set-Up 7

3.2 Brane-Anti-Brane Pair 10

3.3 Branes at Angles 11

4. Inflation 13

4.1 General Set-Up 13

4.2 Hypercubic Compactification 15

4.3 The String Scale 17

4.4 Generic Compactification 18

4.5 Reheating and Defect Production 20

5. Discussions 22

6. Summary and Remarks 23

A. Summing Over The Lattice 24

\section{Introduction}

By now, the inflationary universe [1] is generally recognized to be the most likely scenario that explains the origin of the big bang. So far, its predictions of the flatness of the universe and the almost scale-invariant power spectrum of the density perturbation that seeds structure formations are in very good agreement with the cosmic microwave background (CMB) observations [2, 3]. In standard inflationary models [4], the physics lies in the inflaton potential. However, conventional physics (such as the standard electroweak and strong interaction model or grand unified theories) does not yield an inflaton potential that agrees with observations (such as enough number of e-foldings, the amplitude of the density perturbation etc.). In the past two decades, numerous phenomenological potentials that give the correct inflationary properties have been proposed, most with little input from fundamental physics. In general, a potential that yields enough e-foldings and the correct magnitude of density perturbation requires some fine-tuning. However, such fine-tuning is in general not preserved by quantum corrections. Recently, the brane inflationary scenario [5] was proposed, where the inflaton is identified with an inter-brane separation, while the 
inflaton potential emerged from brane interactions that is well-studied in string theory [6]. In particular, exchanges of closed string (bulk) modes between branes (the lowest order of which can be determined by the one-loop partition function of the open string spectrum) determine the form of the inflaton potential during the slow-roll epoch. Inflation ends when the branes collide, heating the universe that starts the big bang. This visualization of the brane dynamics allows one to implement inflation physics pictorially.

The brane inflation scenario may be realized in a variety of ways [7, 8, 9, 10]. One possibility is the brane-anti-brane interaction and annihilation [7, 8]. Although this potential is too steep for inflation, a particularly appealing scenario proposed by Burgess etc. [8] shows that a hypercubic compactification of extra dimensions flattens the potential to yield enough e-foldings for a viable inflationary epoch. The brane inflationary scenario for branes at a fixed angle is studied by Garcia-Bellido etc. [9], which is a generalization of the brane-anti-brane scenario and is quite natural in string-model building. We re-analyze the brane inflationary scenario by combining and generalizing these two observations and find that the result is very robust. For cases where inflation is generic, we use the CMB data to determine the string scale to be $M_{s} \lesssim M_{G U T}$.

From experimental data and assuming MSSM, the gauge couplings of the standard model unify at the GUT scale [11]:

$$
\alpha(G U T) \simeq 1 / 25 \quad M_{G U T} \simeq 2 \times 10^{16} \mathrm{GeV}
$$

In the brane world scenario, the standard model particles are open string (brane) modes while graviton and other closed string modes are bulk modes. This may be naturally realized in Type I (or orientifold) string theories, with string scale $M_{s}=1 / \sqrt{\alpha \prime}$ and large compactified dimensions. After compactification to 4-D spacetime, the Planck mass, $M_{P}=$ $\left(8 \pi G_{N}\right)^{-1 / 2}=2.42 \times 10^{18} \mathrm{GeV}$, is given by, via dimensional reduction [12, 13]

$$
g_{s}^{2} M_{P}^{2}=\frac{M_{s}^{8} V}{(2 \pi)^{6} \pi}=\frac{M_{s}^{8} V_{t} V_{P}}{(2 \pi)^{6} \pi}
$$

Here $V=V_{t} V_{P}$ is the 6-D compactification volume, where the $(p-3)$ dimensions of the stack of $\mathrm{D} p$-branes we live in are compactified with volume $V_{t}$. Here $g_{s}=e^{\phi_{D}}$ is the string coupling determined by the dilaton expectation value $\phi_{D}$, which is related to the gauge coupling $\alpha$ via

$$
g_{s}=2 M_{s}^{p-3} V_{t}(2 \pi)^{-(p-3)} \alpha\left(M_{s}\right)=2 v_{t} \alpha\left(M_{s}\right)
$$

The presence of the factor $(2 \pi)^{6} \pi$ in Eq.(1.2) appears because the 10-D Newton's constant $G_{10}$ in Type I string theory is given by [13, 6]

$$
8 \pi G_{10}=\kappa^{2}=\frac{g_{s}^{2}(2 \pi)^{7}}{2 M_{s}^{8}}
$$

In general, $M_{G U T} \geq M_{s}$. Crudely speaking, if $M_{G U T} \simeq M_{s}$, the gauge couplings are unified at the string scale via conventional (renormalization group) logarithmic running. If $M_{G U T}>M_{s}$, then $v_{t}>1$ and the gauge couplings will go through a period of power 


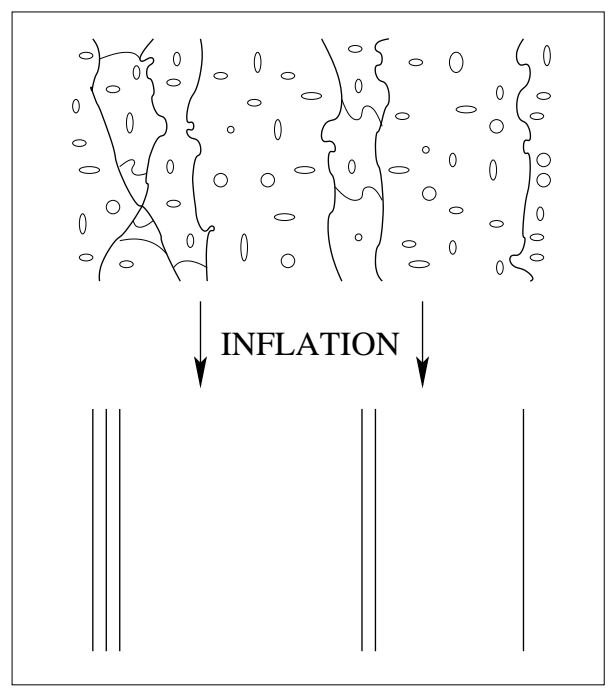

Figure 1: Inflating away brane and bulk fluctuations.

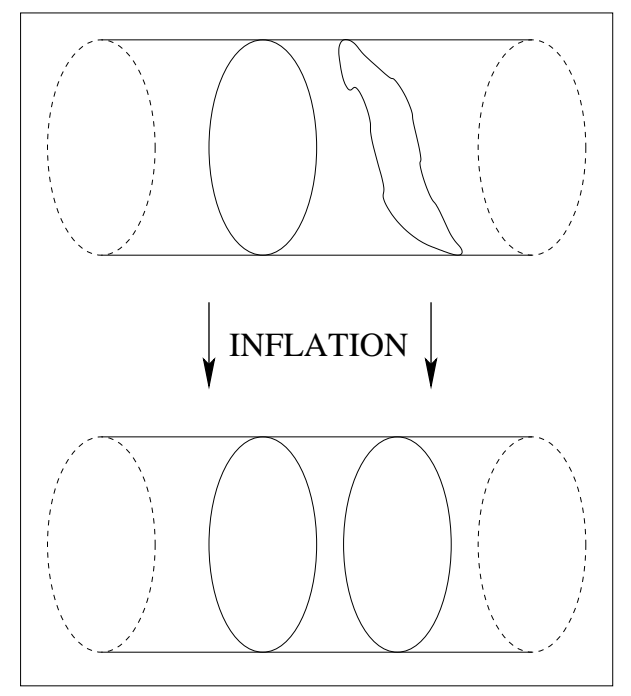

Figure 2: Inflating away non-wrapping angles between branes.

running, unifying at the string scale [14]. However, the present experimental data does not determine the value of $M_{s}$. It can take value anywhere between $1 \mathrm{TeV}$ to $M_{G U T}$ [15].

The key data in the cosmic microwave background (CMB) we use are the density perturbation magnitude measured by COBE [2] and its power spectrum index $n$ [3]

$$
\delta_{H}=1.9 \times 10^{-5} \quad|n-1|<0.1
$$

Our crucial assumptions are that the cosmological constant is negligible after inflation and that the radions (moduli of the compact dimensions) and the dilaton are stabilized by some unknown physics. This assumption precludes the dynamics of these moduli from playing a role in inflation. (Otherwise, the picture becomes much more complicated and modeldependent. We shall briefly discuss how they may play a role in an inflationary scenario 16, 17.)

For two branes at a distance and at angle $\theta$, the parameters coming from string theory are essentially $M_{s}$ and $0<\theta \leq \pi$, as well as the integers $p \geq 3$ and $d_{\perp}$, where $p+d_{\perp} \leq 9$. The generic inflationary picture that emerges in our analysis may be summarized as follows:

- The branes may start out wrinkled and curved, with matter density or defects on them and in the bulk. They may even intersect each other in the uncompactified directions. Generically, there is a density of strings stretched between branes as well. They look like massive particles to the 4-D observer. Branes with zero, one or two uncompactified dimensions are defects that look like point-like objects, cosmic strings and domain walls, respectively. Fortunately, inflation will smooth out the wrinkles, inflate away the curvature and the matter/defect/stretched-string densities, and red shift the intersections exponentially far away from any generic point on the brane, so one may consider the branes with 3 uncompactified dimensions to be essentially parallel in the uncompactified directions, with empty branes and empty bulk. 
- Generically the inflaton potential for a brane-anti-brane system is too steep for inflation [7, 8]. Ref. [8] shows that in a hypercubic toroidal compactification, the images of the brane also exert forces on the anti-brane. For separations comparable to the compactification size, the forces tend to cancel. This softens the potential so there may be enough inflation. If the branes start out far enough apart, there will be a slow-roll period for inflation before they collide. It is sensible to ask what is the probability $P$ of sufficient inflation if the brane and the anti-brane are randomly placed inside the compactified volume. Depending on the number $d_{\perp}$ of large extra dimensions, we find the probability $P \sim(3 \%)^{d_{\perp}}$ This means that brane-anti-brane inflation is not very likely.

- We find that hypercubic compactification is not necessary. For the same brane interaction and compactification volume, we find that $P$ for a generic compactification is comparable to that for a hypercubic toroidal compactification. This point is illustrated with a rectangular torus.

- Ref.[9] argues that branes at angles improves the situation. A D $p$-brane differs from an anti-D $p$-brane only by an opposite Ramond-Ramond (R-R) charge. This charge corresponds to an orientation, so the special case when the angle $\theta=\pi$ corresponds to the brane-anti-brane case. Two branes at generic angle $\theta$ collide to form a lower energy brane (while brane-anti-brane annihilates). For two randomly placed branes at an angle, the probability $P$ of sufficient inflation increases to close to unity as the angle becomes small. Besides the factor of $(2 \pi)^{6} \pi$ in Eq.(1.2) and the inclusion of the compactification effect, our analysis differs from Ref.[9] in the choice of the vacuum energy during inflation. Also, we believe it is more natural to choose the rectangular torus (maybe together with a large winding number) to obtain a small $\theta$. In this case, $P \propto \theta^{-d_{\perp}}$. So, for $\theta \lesssim 1 / 10$ and generic compactification, $P$ is of order unity. This implies that inflation is quite generic with a relatively small $\theta$. As $\theta$ becomes smaller, the compactification lattice effect becomes less important. For small enough angle (i.e., $\theta \lesssim 10^{-2}$ ), the compactification lattice effect is negligible. In this case, $P(\theta)$ is very close to unity.

- The magnitude of the density perturbation $\delta_{H}$ (1.5) may be used to fix the string scale. Generically, we have $M_{s} \lesssim M_{G U T}$. In a brane inflationary scenario suggested by the analysis and generic orientifold construction, we find that $M_{s} \sim M_{G U T}$. It will be very interesting to see how close $M_{s}$ is to $M_{G U T}$ in a more realistic string model.

- Soon after inflation ends, the ground state open string modes become tachyonic, which leads to tachyon condensation and brane annihilation/collision, reheating the universe. This is an explicit realization of hybrid inflation 18]. It is well-known that $p$-brane collision generically allows the production of lower-dimensional $(p-2 k)$ branes. Since the inflaton is different from the tachyon modes that are responsible for defect production, the production of defects is generically a serious problem. We argue that the dangerous productions of domain walls and monopole-like defects 
are actually absent, while the production of the more palatable cosmic strings may happen via the Kibble mechanism. In fact, the production of cosmic strings can be considered as a prediction of this brane inflationary scenario. A more careful analysis is clearly needed to determine the cosmic string density.

Generically, bulk modes have gravitational strength couplings and so brane modes are more likely inflaton candidates. In string theory, vacuum expectation values of such scalar brane modes are simply brane separations, which are the inflatons in brane inflation. Compactification must take place and it always softens the inflaton potential to improve $P$ for inflation. Branes at angles is more generic than a brane-anti-brane pair, and a small $\theta$ is easily realized if two compactification sizes have a ratio $\sim \theta$. These lead us to argue that brane inflation is probably quite generic. In this sense, brane interaction may be considered as an explanation of the origin of inflation.

The attractive force between two branes is stronger for smaller separation and larger angle $\theta$. If the early universe starts with a number of branes (as will be the case if the universe starts out to be very hot, or if there is a higher-dimensional brane-anti-brane pair or a non-BPS brane), probably the universe goes through multiple inflationary epochs, with the last collision involving two branes that start out relatively far apart and at small angle. The inflation we have been discussing is the epoch just before their collision. It is interesting to study this scenario in greater detail.

In Section 2, we write down the effective theory for multi-branes. In Section 3, we set up the brane world scenario just before the final inflation takes place, for a brane-anti-brane pair and for two branes at a fixed angle. The application of this brane pair to inflation is discussed in Section 4, where we find that two branes at a small angle in a compactified volume generically generates inflation. We also discuss the issues of reheating and possible defect productions after inflation. In Section 5, we commend on the bulk modes and the robustness of $M_{s} \lesssim M G U T$. The summary and remarks are presented in Section 6 . An appendix contains the details of the compactification lattice effect on the inflaton potential by summing over the images of a brane.

\section{Effective Action for Brane-Stacks}

We write the world-volume action for $N$ parallel BPS D $p$-branes and $M$ anti-D $p$-branes with $d$ large extra dimensions. This action contains all the salient features of the more general effective action which are important in the brane cosmology model: the "slow-roll" potential for the inflaton (brane separation) coming from the combined R-R and NS-NS sectors, multiple and non-commuting scalar fields, and a set of scalar fields which become tachyonic at the end of inflation (or possibly after the slow roll phase has ended). The effective action is expanded about a flat background, with metric $\eta=\operatorname{diag}(-1,1, \ldots)^{1}$ :

$$
S=-\tau_{p} \int d^{p+1} \sigma \quad\left\{\operatorname{tr}_{N}\left[\frac{1}{2} D_{\mu}^{+} \phi^{I} D^{+\mu} \phi^{I}+\frac{1}{4}\left(F^{+}\right)^{2}\right]+\operatorname{tr}_{M}\left[\frac{1}{2} D_{\mu}^{-} \tilde{\phi}^{I} D^{-\mu} \tilde{\phi}^{I}+\frac{1}{4}\left(F^{-}\right)^{2}\right]\right.
$$

\footnotetext{
${ }^{1}$ The effects of the curved background due to the branes is taken into account by a doubling of the R-R sector force.
} 


$$
\left.+\frac{1}{2} \operatorname{tr}_{N}\left[D_{\mu}^{ \pm} T D^{ \pm \mu} T^{\dagger}\right]+V(\phi, \tilde{\phi}, T)\right\}
$$

The brane tension is $\tau_{p}=\alpha^{-(p+1) / 2} / g_{s}(2 \pi)^{p}$, the $N$ brane coordinates, $\phi^{I=1, \ldots, d}$, are scalars in the adjoint of $U(N)$ written as $N \times N$ matrices in the fundamental representation, $\tilde{\phi}^{I}$ are $M \times M$ scalars in the adjoint of $U(M)$ representing the anti-brane coordinates. $T$ is in the $(\mathbf{N}, \overline{\mathbf{M}})$ of $U(N) \times(M)$ 20] and $F^{ \pm}$are the field strengths of $A^{ \pm}$, the $U(N)$ and $U(M)$ connections on the brane and anti-brane stacks in the fundamental representations. The gauge covariant derivatives are

$$
\begin{aligned}
D_{\mu}^{+} \phi^{I} & =\partial_{\mu} \phi^{I}+\frac{i}{2 \pi \alpha^{\prime}}\left[A_{\mu}^{+}, \phi^{I}\right], \\
D_{\mu}^{-} \tilde{\phi}^{I} & =\partial_{\mu} \tilde{\phi}^{I}+\frac{i}{2 \pi \alpha^{\prime}}\left[A_{\mu}^{-}, \tilde{\phi}^{I}\right], \\
D_{\mu}^{ \pm} T & =\partial_{\mu} T+\frac{i}{2 \pi \alpha^{\prime}}\left(A_{\mu}^{+} T-T A_{\mu}^{-}\right),
\end{aligned}
$$

The potentials are given by

$$
\begin{aligned}
V(\phi, \tilde{\phi}, T) & =V_{b}(\phi, \tilde{\phi})+V_{s}(\phi, \tilde{\phi}, T)+V_{T}+V_{l}(\phi, \tilde{\phi}), \\
V_{b} & =-\frac{1}{4\left(2 \pi \alpha^{\prime}\right)^{2}}\left\{\operatorname{tr}_{N}\left(\left[\phi^{I}, \phi^{J}\right]\left[\phi^{I}, \phi^{J}\right]\right)+\operatorname{tr}_{M}\left(\left[\tilde{\phi}^{I}, \tilde{\phi}^{J}\right]\left[\tilde{\phi}^{I}, \tilde{\phi}^{J}\right]\right)\right\}, \\
V_{s} & =\frac{1}{2\left(2 \pi \alpha^{\prime}\right)^{2}} \operatorname{tr}_{N}\left[\phi^{I} T T^{\dagger} \phi^{I}+T \tilde{\phi}^{I} \tilde{\phi}^{I} T^{\dagger}-2 \phi^{I} T \tilde{\phi}^{I} T^{\dagger}\right], \\
V_{T} & =-\frac{1}{4 \alpha^{\prime}} \operatorname{tr}_{N}\left(T T^{\dagger}\right)+O\left(T^{4}\right), \\
V_{l} & =(N+M)-\sum_{n k} \frac{\hat{\beta} g_{s}^{2} \alpha^{\prime 4} \tau_{p}}{\left[\sum_{I}\left(\phi_{n n}^{I}-\tilde{\phi}_{k k}^{I}\right)^{2}\right]^{(d-2) / 2}},
\end{aligned}
$$

where $\hat{\beta}=2^{5} \pi^{7-d / 2} \Gamma((d-2) / 2)$. The potential $V_{b}$ is obtained from the non-Abelian generalization of the Dirac Born-Infeld action [21. The form of the tachyon scalar interactions, $V_{s}$, can be easily deduced when $N \neq M$, these being the only quartic interactions allowed. The coefficients can be deduced by specializing to the $N=M=1$ case, and using the fact that the tachyon quadratic term goes negative for $(\phi-\tilde{\phi})^{2}<2 \pi^{2} \alpha^{\prime}$ [22]. Alternatively these terms appear as the T-duals of the tachyon gauge-kinetic terms.

The tachyon potential, $V_{T}$, can been calculated by various means [23, 24, 25]:

- The result from level truncated cubic string field theory [23, 24] gives a tachyon mass ${ }^{2}$ which agrees with that of the NS open string tachyon, $-\frac{1}{2 \alpha^{\prime}}[6]$; this is the mass of the perturbative state which appears in the brane-brane system upon reversal of the $\mathrm{R}-\mathrm{R}$ charge of one.

- The tachyon potential obtained from boundary string field theory (BSFT) [25] is not in canonical form, and a field redefinition to its canonical form will involve all components of the string field. BSFT does however have the advantage that it clearly verifies Sen's conjecture [26] that at the minimum of the tachyon potential, the physics is that of the closed string vacuum. 
- The $T^{4}$ term in $V_{T}$ has been calculated at the lowest level in cubic string field theory for $N=M=1$ to be $+\frac{1}{2 \alpha^{2}} T^{4}$ [23], but there is ambiguity in its form in general, with both $\operatorname{tr}\left(T^{4}\right)$ and $\left[\operatorname{tr}\left(T^{2}\right)\right]^{2}$ terms allowable; BSFT predicts only the former term [25], but it is uncertain whether this holds under the field redefinition.

- For the purposes here, the $T^{2}$ term is most important, since its coefficient dictates the separation at which $T$ becomes tachyonic, ending inflation. ${ }^{2}$ In generalizing this action to that of branes at angles, the coefficient of $T^{2}$ term should become [6] $-\frac{\theta}{4 \pi \alpha^{\prime}}$, where $\theta$ is the angle between the brane-stacks; $\theta=\pi$ reverts to the brane anti-brane case above. The effects of placing the branes at angles is to lessen the distance between the branes at which $T$ becomes tachyonic.

- The presence of the tachyonic modes originates from the matrix nature of the brane positions. This non-commutative property has interesting cosmological consequences. The non-trivial vacuum structure of the tachyon condensate allows the creation of lower-dimensional branes. Since the tachyons are not the inflaton, lower-dimensional brane production will take place after inflation, typically via the Kibble mechanism. It is important that this does not re-introduce the old monopole problem.

Finally, the potential $V_{l}$ takes into account the R-R and NS-NS backgrounds generated by the brane and anti-brane stacks [27, 22]. $\phi_{l n}^{I}$ is the $\ln$ component of $\phi^{I}$, and $V_{l}$ is a function of $\phi_{n n}^{I}-\tilde{\phi}_{k k}^{I}$ only. For $d=2$, the Coulomb-like potential is replaced by a log. This is twice the Chern-Simons brane action, written with the explicit expression for the pull-back of the space-time $p+1$ form potential [27] generated by the branes. Only the term from the massless modes in the expansion of the exact potential is necessary for our purposes, to give the long-distance potential between the brane stacks, before the $T$ fields become tachyonic, and inflation ends. We have doubled the R-R potential to account for the equal force supplied by the NS-NS sector. When we consider branes at angles, the coefficient of $V_{l}$ acquires a factor of $\tan (\theta / 2) \sin ^{2}(\theta / 2)$ [6, 28], with more complicated angular factors for more than one tilted direction; as we shall see, some brane inflation scenarios require branes at small angles to achieve sufficient e-foldings.

\section{Braneworld at the Start of Inflation}

\subsection{Set-Up}

Realistic string models have 6 of the 9 space dimensions compactified. Consider $D p$ branes in 10-dimensional space-time, where $(p-3)$ dimensions parallel to the brane are compactified with volume $V_{\|}$and the $d$ dimensions orthogonal to the brane are compactified with volume $V_{d}$. The remaining 3 spacial dimensions of the $D p$-brane are uncompactified; they span our observable universe. Let $M_{s}$ be the string scale $M_{s}^{-2}=\alpha^{\prime}$. So $4+(p-3)+$ $d=10$ and $V=V_{d} V_{\|}$. In general, the branes in the early universe that will eventually

\footnotetext{
${ }^{2}$ Were we to use the BSFT mass ${ }^{2}$ of $-1 / 4 \ln 2 \alpha^{\prime}$, the end of the inflationary phase would be changed little.
} 
collide after inflation but before the big bang are different from those present in today's braneworld, so $V_{\|}$can be quite different from $V_{t}$ in Eq.(1.2). To simplify the discussion, we shall assume, unless pointed out otherwise, that they largely overlap.

In string theory, there is a T-duality symmetry, i.e., physics is invariant under a Tduality transformation. For toroidal compactification, we have $V=\prod_{i} l_{i}=\prod_{i} 2 \pi r_{i}$, where $l_{i}$ is the size of the $i$ th torus/circle and $r_{i}$ the corresponding radius, $l_{i}=2 \pi r_{i} \cdot{ }^{3}$ If any of the $r_{i}$ is much smaller than the string scale, i.e., $M_{s} r_{i}<1$, the T-dual description is more appropriate :

$$
g_{s} \rightarrow \frac{g_{s}}{r_{i} M_{s}}, \quad r_{i} \rightarrow \frac{1}{r_{i} M_{s}^{2}}
$$

In this dual picture, the new $r_{i} M_{s}$ of the dual $T_{i}$ torus is always larger than or equal to unity. Under this duality transformation, the Dirichlet and Neumann boundary conditions of the open strings are interchanged, and so the branes are also mapped to other types of branes. This allows us to consider only the cases where $M_{s} r_{i} \geq 1$, with $M_{s} r_{i}=1$ the self-dual point.

- Perturbative string theory does not seem to stabilize the dilaton. So we expect dilaton stabilization to arise from non-perturbative stringy effects. Very strongly coupled string theory presumably has a weak dual description, where the dilaton is again not stabilized. This leads us to conclude that the string coupling generically is expected to be $g_{s} \gtrsim 1$. To obtain a theory with a weakly coupled sector in the low energy effective field theory (i.e., $\alpha(G U T)$ is small), it then seems necessary to have the brane world picture [29]. Let us consider the $p>3$ case. In this case,

$$
g_{s}=2\left(M_{s} r_{\|}\right)^{p-3} \alpha\left(r_{\|}\right)=2 v_{\|} \alpha\left(r_{\|}\right)
$$

where $v_{\|}=\left(M_{s} r_{\|}\right)^{p-3}$ and $\alpha\left(r_{\|}\right)$is the gauge coupling at the scale $1 / r_{\|}$. All couplings should unify at the string scale. To get a qualitative picture of the impact of a relatively large string coupling, let us take $g_{s} \sim 1$. So $\alpha$ has logarithmic running up to the scale $1 / r_{\|}$and then power-running between $1 / r_{\|}$and $M_{s}$, yielding an $\alpha\left(M_{s}\right) \sim 1$. For $p=5, M_{s} r_{\|} \sim 5$ while for $p=7, M_{s} r_{\|} \sim \sqrt{5}$. So $\alpha\left(r_{\|}\right)$is essentially $\alpha_{G U T}$. Since the renormalization group flow $\beta$ coefficients (of the standard model gauge couplings) for power-running [14] have almost identical convergence properties as that for the logarithmic running in MSSM and the power running is only over a relatively small energy range, the unification of the gauge couplings at the string scale is probably assured.

- Of the $d$ dimensions orthogonal to the brane, suppose only $d_{\perp}$ of them are large. To simplify the discussions, let the 6-D compactification volume $V$ be

$$
V=V_{\|} V_{\perp} V_{\left(d-d_{\perp}\right)}
$$

\footnotetext{
${ }^{3}$ In general, the radius $r_{i}$ here does not necessarily have to be the radius of the $i$ th torus. It is simply a characteristic length scale of the compactified dimension. In the case of a $\mathbf{Z}_{N}$ orbifold, the volume is given by $\prod_{i} v_{i}=\frac{1}{N} \prod_{i}\left(2 \pi R_{i}\right)^{2} \equiv \prod_{i}\left(2 \pi r_{i}\right)^{2}$. In general, it stands for the first KK mode, i.e., at $r_{i}^{-1}$.
} 
where the $\left(d-d_{\perp}\right)$-D volume $V_{\left(d-d_{\perp}\right)}$ is $\left(2 \pi / M_{s}\right)^{d-d_{\perp}}$ (i.e., at the self-dual value). We shall consider $d_{\perp}+p \leq 9$. Unless pointed out otherwise, we shall assume all the large extra dimensions to have equal size $l_{i}=\ell_{\perp}$ or $r_{i}=r_{\perp}\left(\ell_{\perp}=2 \pi r_{\perp}\right)$, so the remaining dimensions orthogonal to the brane have sizes such that $M_{s} r_{i}=1$. For branes at angle and $p>3$, we shall consider $d_{\perp}$ to range from 2 to 4 .

Note the effect of a larger $V_{d-d_{\perp}}$ is equivalent to an increased $d_{\perp}$, so the physics will simply interpolate between the values considered. This allows us to use the volumes $V_{\perp}=\left(2 \pi r_{\perp}\right)^{d_{\perp}}$ (transverse to the brane) and $v_{\|}$(along the brane) as parameters to be determined. Following from dimensional reduction where the 10-D Newton's constant $G_{10}$ is given by Eq.(1.4), we have

$$
g_{s}^{2} M_{P}^{2}=M_{s}^{2}\left(M_{s} r_{\perp}\right)^{d_{\perp}} v_{\|} / \pi
$$

Note that the factor of $(2 \pi)^{6} \pi$ in $\operatorname{Eq}(3.4)$ is missing in Ref. [7, 8, 9]. The string coupling is $g_{s}=e^{\phi_{D}}$ where $\phi_{D}$ is the dilaton. The BPS D $p$-brane has brane tension $\tau_{p}$ and R-R charge $\mu_{p}$ :

$$
\tau_{p}=\frac{M_{s}^{p+1}}{(2 \pi)^{p} g_{s}}, \quad \mu_{p}=g_{s} \tau_{p}
$$

while an anti-D $p$-brane has the same tension $\tau_{p}$ but opposite $\mathrm{R}-\mathrm{R}$ charge. These relations are subject to quantum corrections, which we shall ignore for the moment.

We shall demand that $M_{s} r_{\perp} \gg 1$ and $d_{\perp}>1$. For instance, this is required within string theory if we want to treat the bulk dynamics using only the low-energy effective field theory corresponding to the massless string states. These are also the conditions under which the nonlinear contributions of Einstein's equations are negligible when considering the gravitational field of a single $D$-brane, say. Therefore our approximate $4 \mathrm{D}$ effective field theory treatment is self-consistent.

- In principle, we can also consider $d_{\perp}=1$. However, in this case, the potential between branes becomes confining and the presence of branes will induce a warped geometry in the bulk [30]. As a result of this, the physics is quite different and will not be considered here.

- The standard model of strong and electroweak interactions requires at least $5 \mathrm{Dp}$ branes. Suppose today's universe is described by a Type I or an orientifold string model, which is supersymmetric at scales above the electroweak scale (or non-supersymmetric but with a very small cosmological constant). Suppose the universe starts out with more branes than its vacuum state. This may happen if the early universe is very hot, which probably is a hot gas of different $p$-branes oriented randomly. Alternatively, the universe may start out with some brane-anti-brane pair or non-BPS branes [19, 26]. This set-up may emerge without a very hot universe. Eventually, the branes will move and collide, until it reaches the brane configuration of the vacuum state; that is, all branes except those in the ground state of the string model disappear, via collisions, decays and/or annihilations. Before the last collision, we 
want to see if inflation happens, and how generic inflation is. If the branes that will collide are randomly placed in the compactification volume, we want to estimate the probability $P$ that sufficient inflation will take place before collision. The universe will then reheat to originate the big bang. When this happens, the magnitude of the density perturbation will be used to fix the string scale. It is important to see if the density perturbation power spectrum index satisfies the observational bound.

\subsection{Brane-Anti-Brane Pair}

Suppose that inflation takes place before the last $D p$-brane-anti- $D p$-brane pair annihilates. We may also consider the case of a stack of parallel $N D p$-branes and a stack of $M$ parallel anti- $D p$-branes. The 4 -D inflaton potential has a term due to brane tension and a term due to the interactions between the branes. The force between stationary parallel BPS $D p$-branes is zero, due to the exact cancellation between the attractive NS-NS and the repulsive Ramond-Ramond (R-R) couplings. On the other hand, the potential between a $D p$-brane and an anti- $D p$-brane is attractive, since the $\mathrm{R}-\mathrm{R}$ coupling is also attractive in this case. To simplify the problem, let us take $N=M=1$, i.e., a single anti- $D p$-brane. Inflation takes place when the last anti- $D p$-brane is approaching a $D p$-brane and inflation ends when it annihilates with the brane. The universe is taken to be supersymmetric at this scale, so after annihilation, we expect the vacuum energy to be essentially zero, where the remaining brane tensions are exactly canceled by the presence of orientifold planes. We shall consider the simple case where the remaining branes are sitting at the orientifold fixed points (evenly distributed). In this case, the interaction of the anti- $D p$-brane with the remaining branes is exactly canceled by that with the orientifold planes. The 4 -D effective action of a pair of $D p$-anti- $D p$-branes takes the form:

$$
\Gamma \simeq \int d^{4} x \sqrt{|g|}\left(\frac{\tau_{p} V_{\|}}{2}\left(\partial_{\mu} y_{b} \partial^{\mu} y_{b}+\partial_{\mu} y_{a} \partial^{\mu} y_{a}\right)-V(y)+\ldots\right)
$$

where the brane is at $y_{b}$ and the anti-brane is at $y_{a}$, so the separation is $y=y_{b}-y_{a}$. With

effective $\kappa^{2} /\left(V_{\|} V_{d-d_{\perp}}\right)$ where $V_{d-d_{\perp}}=\left(2 \pi / M_{s}\right)^{9-p-d_{\perp}}$, the potential for $d_{\perp}>2$ is easy to write down :

$$
V_{l}(y)=2 \tau_{p} V_{\|}-\frac{\kappa^{2} \beta \tau_{p}^{2} V_{\|}}{y^{d_{\perp}-2}}\left(\frac{M_{s}}{2 \pi}\right)^{9-p-d_{\perp}}
$$

where

$$
\begin{aligned}
\tau_{p} & =M_{s}^{p+1} /(2 \pi)^{p} g_{s}, \\
\beta & =\pi^{-d_{\perp} / 2} \Gamma\left(\left(d_{\perp}-2\right) / 2\right) / 2 \quad d_{\perp}>2
\end{aligned}
$$

and $\kappa^{2}$ is given in Eq.(1.4). Here $\beta / 2$ comes from the inverse surface area from Gauss's Law and the extra factor of 2 in $\beta$ is due to the sum of the NS-NS and the R-R couplings. The potential becomes logarithmic and $\beta=1 / \pi$ for $d_{\perp}=2$. 


\subsection{Branes at Angles}

String models with branes at angles can be quite realistic [31]. Following Ref. [9], let us consider a supersymmetric string model (or a non-supersymmetric model with a zero cosmological constant) where a brane wrapping 1-cycles in a two dimensional torus. Let the two torus have radii $r_{\|}$(or size $\ell_{\|}=2 \pi r_{\|}$) and $u r_{\|}$, where $u<1$. This brane wraps a straight line in the $\left\{n_{f}[a]+m_{f}[b]\right\}$ homology class. The energy density of this brane is just [6]

$$
E_{f}=\tau_{4} \ell_{\|}\left[\sqrt{n_{f}^{2}+\left(u m_{f}\right)^{2}}\right]
$$

This energy is of course precisely canceled by the presence of other branes and orientifold planes in the model. Suppose this brane results from the collision of two branes in the early universe, each of which wraps a straight line in the $\left\{n_{i}[a]+m_{i}[b]\right\}$ homology class. Since the total (homological) charge is conserved, $\left(m_{f}, n_{f}\right)=\left(m_{1}+m_{2}, n_{1}+n_{2}\right)$. The energy density of the two-brane system is

$$
E_{2}=\tau_{4} \ell_{\|}\left[\sqrt{n_{1}^{2}+\left(u m_{1}\right)^{2}}+\sqrt{n_{2}^{2}+\left(u m_{2}\right)^{2}}\right] .
$$

So the energy density before the brane collision, up to an interaction term, is

$$
V_{0}=E_{2}-E_{f}
$$

Let us consider the case where $V_{0}>0$. Before collision, the branes are at an angle $\theta=$ $\phi_{1}-\phi_{2}$, where $\tan \phi_{i}=\left(u m_{i} / n_{i}\right)$ and are separated by a distance in directions orthogonal to the torus. When this separation between the branes approaches zero (see Figure 3), the collision results in the $\left(m_{f}, n_{f}\right)$ brane. In the case where $\left(m_{f}, n_{f}\right)=(0,0)$, with non-zero $\left(m_{i}, n_{i}\right)$, we have a brane-anti-brane pair (with $\theta=\pi$ ) which annihilate when they collide. When $\left(m_{f}, n_{f}\right)$ is non-zero, a brane is left behind. If $m_{f}$ and $n_{f}$ are co-prime, this brane is at an angle. Such branes appear naturally in many phenomenologcally interesting models [31. If $m_{f}$ and $n_{f}$ have a common factor $k, m_{f}=k \hat{m}_{f}, n_{f}=k \hat{n}_{f}$, we may consider this as $k$ branes in the $\left\{\hat{n}_{f}[a]+\hat{m}_{f}[b]\right\}$ homology class. If either $m_{f}$ or $n_{f}$ is zero, presumably the brane (or branes) is parallel to an orientifold plane. In effective low-energy field theory, this is the Higgs mechanism. Suppose there is a $U(N)$ gauge group associated with each brane before collision (i.e., $\left.n_{1}=n_{2}=N\right)$. Then there is a $U(N) \times U(N)$ gauge symmetry together with a bifundamental scalar, which are open strings stretched between the branes. During

collision, the bifundamental scalar develops a vacuum expectation value and spontaneous symmetry breaking takes place:

$$
U(N) \times U(N) \rightarrow U(N)
$$

Suppose these two branes are separated by a large distance in the other compactified directions. Then the effective potential is given by [6, 28]

$$
V(y)=V_{0}-\frac{\beta M_{s}^{6-d_{\perp}} \sin ^{2} \theta / 2 \tan \theta / 2}{4 \pi y^{d_{\perp}-2}} .
$$

where $V_{0} \propto \tan ^{2} \theta$. For $d_{\perp}=2$, the potential has a logarithmic form. Note that the potential term vanishes as $\theta \rightarrow 0$. In this limit, the branes are parallel and are BPS with 


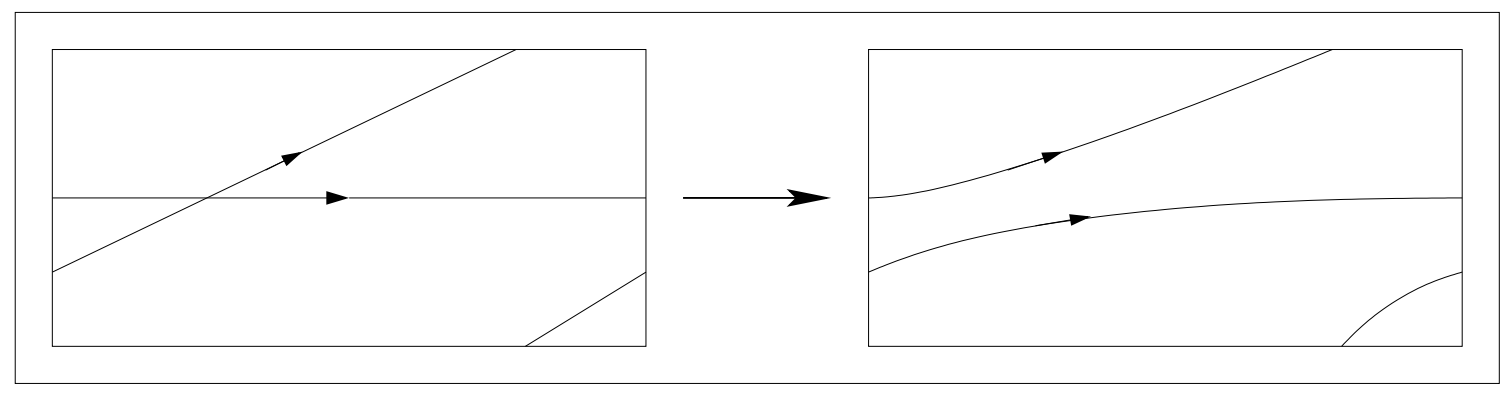

Figure 3: Branes wrapping a torus with sides $\ell_{\|}$and $u \ell_{\|}$, where $u<1$. The two branes $((1,1)$ and $(1,0))$ are at an angle $\theta$, but they are separated in directions orthogonal to the torus. When that separation approaches zero, the two branes collide to become a single $(2,1)$ brane.

respect to each other. In the other limit, as $\theta \rightarrow \pi$, the potential blows up due to the $\tan \theta / 2$ factor. This happens because the brane becomes parallel to the anti-brane and so the strings stretching between them are free to move in that direction. In this case, there is a volume factor to be taken into account. Alternatively, one simply use the potential for the brane-anti-brane system given earlier.

Here, we are interested in the small angle case. To get a qualitative feeling of the scenario, let us take for example $m_{1}=1$ and $m_{2}=0$. Then $\phi_{2}=\arctan \left(u m_{2} / n_{2}\right)=0$, and $\phi_{1}=\arctan \left(u / n_{1}\right) \equiv \theta \ll 1$. This may be achieved with large $n_{1}$ or small $u$, or a combination of both. Small $\theta$ may be realized in a number of ways in string theory :

- We may simply take $n_{1}$ to be very large, the case considered in Ref.[9]. Generically, large $n_{1}$ implies the string model today will have a brane with a very high winding number. This is a possibility that deserves further study.

- Suppose the resulting brane that wraps around $\ell_{\|}$is not among the branes that are responsible for the standard model gauge fields, that is, $\ell_{\|}$is part of $V_{\perp}$ and not part of $V_{\|}$. Then there is hardly any constraint on the value $\ell_{\|}$and we may take $u$ to be very small with $n_{1}=1$, say.

- Generically, the resulting brane will wrap around $\ell_{\|}$, so $\ell_{\|}$is likely to be a part of $V_{t}$ in Eq.(1.2). We may estimate how small $u$ may be. First recall that $u M_{s} \ell_{\|} \geq 2 \pi$. On the other hand, $\ell_{\|}$is bounded by $M_{s} \ell_{\|} / 2 \pi \lesssim v_{\|}$, so, following Eq.(1.3), $u \geq 2 \alpha / g_{s}$. The smallest we can reasonably have is $u \approx 10^{-2}$. Since it is quite reasonable for a string model to have $n_{1} \geq 1$ (to accomodate the standard model, we expect $n_{f} \geq 5$, which implies $n_{1} \geq 4$ in our example), $\theta \simeq u / n_{1} \approx 10^{-2}$ is not unreasonable. As we shall see, $\theta \sim 0.1$ is quite sufficient.

To facilitate comparison with Ref.[9], we may consider the $d_{\perp}=4$ case. In the small angle approximation we have (taking $n_{1}=n_{2}=1$ ),

$$
V(y)=\frac{\tau_{4} \ell_{\|} \tan ^{2} \theta}{4}-\frac{M_{s}^{2} \sin ^{2} \theta / 2 \tan \theta / 2}{8 \pi^{3} y^{2}}
$$




\section{Inflation}

\subsection{General Set-Up}

The inflaton as seen by a 4-D observer

$$
\Gamma \simeq \int d^{4} x \sqrt{|g|}\left(\frac{1}{2} \partial_{\mu} \psi \partial^{\mu} \psi-V(\psi)+\ldots\right)
$$

is related to the brane separation $y=y_{b}-y_{a}$ in Eq.(3.6) by:

$$
\psi=y \sqrt{\frac{\tau_{p} V_{\|}}{2}}=y M_{s}^{2} \sqrt{\frac{v_{\|}}{2 g_{s}(2 \pi)^{3}}}
$$

where $v_{\|}=\left(M_{s}^{p-3} V_{\|}\right) /(2 \pi)^{p-3}$. So the effective potential for the inflaton becomes, for $d_{\perp}>2$ and $d_{\perp}=2$, respectively,

$$
\begin{aligned}
& V(\psi)=A\left(1-\frac{\lambda}{\psi^{d_{\perp}-2}}\right) \\
& V(\psi)=A\left(1+\lambda \ln \left(\psi / M_{s}\right)\right)
\end{aligned}
$$

where $A$ and $\lambda$ are given by Eq.(3.7) for the brane-anti-brane case and by Eq.(3.11) for the branes at angle case. For small $\theta$ and $d_{\perp}=4$, we shall use Eq.(3.12).

The equation of motion for $\psi$ is

$$
\ddot{\psi}+3 H \dot{\psi}+V^{\prime}=0
$$

where the prime indicates derivative with respect to $\psi$. For inflation to take place, there is some choice of $\psi$ so that the potential $V(\psi)$ satisfies the slow-roll conditions $\epsilon<<1$ and $|\eta|<<1$, where :

$$
\begin{aligned}
& \epsilon=\frac{1}{2} M_{P}^{2}\left(\frac{V^{\prime}}{V}\right)^{2} \\
& \eta=M_{P}^{2} \frac{V^{\prime \prime}}{V}
\end{aligned}
$$

During the slow-roll epoch, $\ddot{\psi}$ in Eq. (4.4) is negligible and the Hubble constant $H$ is given by

$$
H^{2} \simeq\left(\frac{\dot{a}}{a}\right)^{2}=\frac{V}{3 M_{P}^{2}}
$$

where $a(t)$ is the cosmic scale factor, and we may relate the value of $\psi_{N}$ as a function of the number $N_{e}$ of e-foldings before the end of inflation. The value of $\psi_{\text {end }}$ is determined when the slow-roll condition breaks down, when either $|\eta| \approx 1$ or $\epsilon \approx 1$. Generically, $|\eta|>>\epsilon$ in brane inflationary scenarios. It takes $N_{e}$ e-foldings for $\psi_{N}$ to reach $\psi_{\text {end }}$.

$$
N_{e}=\int_{t_{N}}^{t_{e n d}} H d t=\int_{\psi_{N}}^{\psi_{\text {end }}} H \frac{d \psi}{\dot{\psi}}=\frac{1}{M_{P}^{2}} \int_{\psi_{\text {end }}}^{\psi_{N}} \frac{V}{V^{\prime}} d \psi
$$


Inflation may also end when the ground state open string mode becomes tachyonic:

$$
m_{T}^{2}=\left(\frac{M_{s}^{2} y}{2 \pi}\right)^{2}-\frac{M_{s}^{2} \theta}{2 \pi}
$$

However, in the scenarios we are considering, the slow-roll condition breaks down before the tachyon mode appears. As we shall see, this has a non-trivial impact on defect productions after inflation.

We know that the density perturbation measured [2] from Cosmic Microwave Background $(\mathrm{CMB})$ is $\delta_{H}=1.9 \times 10^{-5}$. This is the density perturbation at $N_{e}$ e-foldings before the end of inflation, where

$$
N_{e} \simeq 60+\frac{2}{3} \ln \left(M_{s} / 10^{16} G e V\right)+\frac{1}{3} \ln \left(T_{R H} / 10^{14} G e V\right)
$$

where $T_{R H}$ is the reheating temperature after inflation. Here, for $d_{\perp} \geq 2$,

$$
N_{e}=\frac{d_{\perp}-1}{d_{\perp}}\left[\frac{y_{N}}{y_{e n d}}\right]^{d_{\perp}}
$$

The power index of the density perturbation

$$
n-1=2 \eta-6 \epsilon
$$

For $\epsilon \ll \eta$, the spectral index $n$ and its variation with respect to the wavenumber $k$ are given by:

$$
\begin{gathered}
n-1=\left.2 \eta\right|_{N_{e}} \approx-\frac{2\left(d_{\perp}-1\right)}{d_{\perp} N_{e}} \\
\frac{d n}{d \ln k}=\left.2 \xi^{2}\right|_{N_{e}}=2 M_{p}^{4} \frac{V^{\prime} V^{\prime \prime \prime}}{V^{2}} \approx \frac{2\left(d_{\perp}-1\right)}{d_{\perp} N_{e}^{2}}
\end{gathered}
$$

In our case, we have

$$
\begin{aligned}
\eta & =-\beta\left(d_{\perp}-1\right)\left(d_{\perp}-2\right)\left(\frac{\ell_{\perp}}{y}\right)^{d_{\perp}} \quad d_{\perp}>2 \\
& =-\frac{1}{\pi}\left(\frac{\ell_{\perp}}{y}\right)^{2} \quad d_{\perp}=2
\end{aligned}
$$

Generically, we need $|\eta| \lesssim 1 / 60$ during the early inflationary stage. Since $\left|y_{i}\right|<\ell_{\perp} / 2$, we see that $\eta$ can never be small enough to satisfy the slow-roll condition. This is noted in Ref. [7, 8].

Now let us consider the more general situation of branes at angle. As pointed out in Ref. [9], the situation improves. For small $\theta$ and $n_{1}=1$, we have $\theta \simeq u / n_{1}=u$. With $(2 \pi)^{d_{\perp}+2} \pi g_{s}^{2} M_{P}^{2}=M_{s}^{2}\left(M_{s} \ell_{\|}\right)\left(M_{s} u \ell_{\|}\right)\left(M_{s} \ell_{\perp}\right)^{d_{\perp}}$,

$$
\begin{aligned}
\eta & \approx-\frac{3 \theta^{2}}{2 \pi^{2}}\left(\frac{\ell_{\perp}}{y}\right)^{4} & d_{\perp}=4 \\
& \approx-4 \pi \theta^{2}\left(\frac{\ell_{\perp}}{y}\right)^{2} & d_{\perp}=2
\end{aligned}
$$


To have enough number of e-foldings of slow-roll while the branes are separated, we get a bound on $\theta$ by requiring $|\eta| \leq 1 / 60$ at the beginning of inflation. However, when $|y| \sim \ell_{\perp} / 2$, the compactification effect is important and Eq.4.15) for $\eta$ is not valid (see the discussion below). For the compactification effect to be negligible so Eq.4.15) is valid, a smaller $\theta$ is needed, say

$$
\theta<10^{-2}
$$

With such a small $\theta$, the probability $P$ of sufficient inflation approaches unity. A small but not too small value of $\theta$ will still increase $P$ and further improves the naturalness of existence of an inflationary epoch in the brane world.

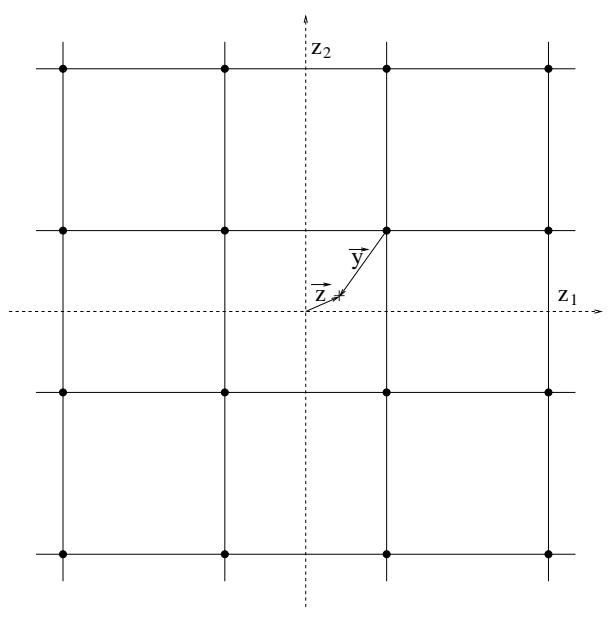

Figure 4: Compactification on hypercubic lattice (solid lines), where one brane sits at the lattice points while the other brane is at $z$. Here, $z$ (and $\varphi$ ) are mea(the intersection of the dashed lines). sured with respect to the antipodal point

\subsection{Hypercubic Compactification}

When the brane separation is comparable to the lattice size, we must include the forces exerted by the images of one brane on the other brane. (The net force on a brane due to its own images is exactly zero.) This always tend to soften (or flattens) the potential. Let us first consider the hypercubic lattice for the brane-anti-brane pair discussed in Ref.[8]. Let the brane sit at the origin $\vec{y}=\overrightarrow{0}$, while we measure the position of the anti-brane from the antipodal point $\left(\ell_{\perp} / 2, \ell_{\perp} / 2, \ldots\right)$, at which the net force on the anti-brane is zero : $z_{i}=y_{i}-$ $\ell_{\perp} / 2$ and define the corresponding $\varphi_{i}=\psi_{i}-\psi_{i} *$ where $\psi_{i} *$ is the value of $\psi_{i}$ at the antipodal point. (See Figure 4.) The potential for the D-dimensional hypercubic lattice is given by:

$$
V(\vec{y})= \begin{cases}A-B \sum_{i} \frac{1}{\left|\vec{y}-\vec{r}_{i}\right|^{d_{\perp}-2}}, & d_{\perp}>2 \\ A+B \sum_{i} \ln \left|\vec{y}-\vec{r}_{i}\right|, & d_{\perp}=2\end{cases}
$$

where the sum is over all the lattice sites $\vec{r}_{i}$ (positions of the images of the brane), where $\vec{r}_{0}$ is at the origin $\vec{y}=\overrightarrow{0}$. In order to estimate the potential around the antipodal point, we expand to 4 th order in $\vec{z}$ (or $\varphi$ ) around the center of the elementary cell, i.e., $\vec{z}=\overrightarrow{0}$. Summing over the images of the other brane for hypercubic lattices (see Appendix for details), we have, for small $\varphi$,

$$
V(\varphi) \simeq A\left(1-\hat{\lambda}\left[D \sum_{i \neq j} \varphi_{i}^{2} \varphi_{j}^{2}-C \sum_{i} \varphi_{i}^{4}\right]\right)
$$

where $\hat{\lambda}$ is related to $\lambda$ by some rescaling,

$$
\hat{\lambda}=\lambda \ell_{\perp}^{-2-d_{\perp}}\left(\frac{\tau_{p} V_{\|}}{2}\right)^{-1-d_{\perp} / 2}
$$




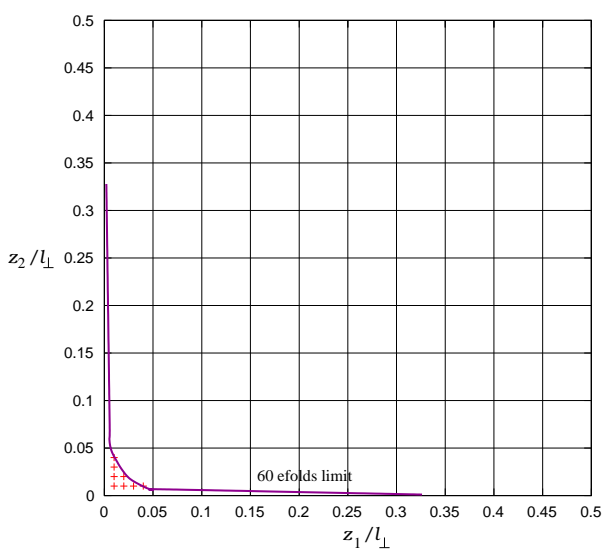

Figure 5: The 60 e-folds region for brane anti-brane interaction. The brane is sitting at the upper right corner. There will be 60 e-folds or more if the anti-brane starts in the region in the lower left corner.

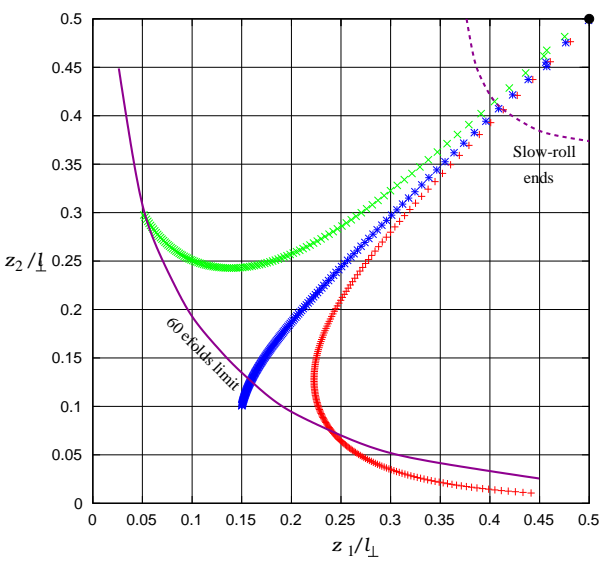

Figure 6: The 60 e-folds region for branes at fixed angle $\theta \sim 1 / 6$. Three possible inflaton paths with enough e-folds are shown. Intervals along each path acts as a clock. Note that the right path starts out with $|\eta| \sim 1$ and then slows down during the inflationary epoch.

and the values of $C$ and $D$ are given in Table 1 . As pointed out in Ref. [8], the quadratic terms in $\varphi$ are absent in hypercubic lattices.

\begin{tabular}{|c|r|c|c|r|r|c|}
\hline$d_{\perp}$ & \multicolumn{1}{|c|}{$\mathrm{C}$} & $\mathrm{D}$ & $\mathrm{F}$ & $F \beta$ & $2 z_{*} / \ell_{\perp}$ & $P(\pi)$ \\
\hline \hline 2 & 3.94 & 23.64 & 15.76 & 5.0 & $3.5 \%$ & $2 \times 10^{-3}$ \\
\hline 3 & 6.22 & 18.64 & 37.26 & 5.9 & $3.3 \%$ & $<10^{-4}$ \\
\hline 4 & 15.41 & 30.82 & 123.3 & 6.2 & $3.1 \%$ & $\sim 10^{-6}$ \\
\hline
\end{tabular}

The inflaton is a multi-component field, so the inflaton path can be quite complicated. Some sample paths are shown in the figures. To get a qualitative feeling of the potential, let us consider the diagonal path

$$
\begin{aligned}
V(\varphi) & \simeq A\left(1-\hat{\lambda} F \varphi^{4}\right) \\
F & =\frac{d_{\perp}\left(d_{\perp}-1\right)}{2} D-d_{\perp} C
\end{aligned}
$$

For large $N_{e}$, we have

$$
\begin{aligned}
\eta & =-12 M_{P}^{2} \hat{\lambda} F \varphi^{2}=-12 F \beta\left(\frac{z}{\ell_{\perp}}\right)^{2} \\
N_{e} & \approx \frac{3}{2|\eta|}
\end{aligned}
$$

The quantum fluctuation in deSitter space $H / 2 \pi$ yields

$$
\delta_{H}^{2}=\left.\frac{1}{75 \pi^{2} M_{P}^{6}} \frac{V^{3}}{\left(V^{\prime}\right)^{2}}\right|_{\varphi_{N}} \approx \frac{32 A \hat{\lambda} F N_{e}^{3}}{75 \pi^{2}}
$$


To have enough time for slow-roll, let us consider the value $z *$ at $|\eta| \approx 1 / 60$, which implies

$$
\frac{2 z_{*}}{\ell_{\perp}} \approx 3 \%
$$

This means the probability $P(\pi)$, which is essentially the fraction of the cell volume that yields 60 or more e-foldings for $d_{\perp} \geq 2$,

$$
P(\pi) \sim\left(\frac{2 z_{*}}{\ell_{\perp}}\right)^{d_{\perp}} \sim(3 \%)^{d_{\perp}}
$$

of enough inflation for an initial randomly placed brane-anti-brane pair is quite small. A more careful estimate of the region with enough inflation in the $d_{\perp}=2$ case is shown in Figure 5 (since the lattice has at least a 4 -fold symmetry, we need to show only a quarter of a lattice cell in the $d_{\perp}=2$ case), while the value of $P(\pi)$ is given in Table 1 . In this case, a fine-tuning on the initial condition seems to be required to obtain enough inflation.

Fortunately, the situation is immensely improved when the branes are at a small $\theta$. Actually, one may argue that, to have a brane-anti-brane pair (that is $\theta=\pi$ ) involves some sort of a fine-tuning because, apriori, randomly placed branes will be at an angle $\theta \neq \pi$. To get a qualitative feeling of the impact of small $\theta$, let us consider a 4-brane pair with $n_{1}=n_{2}=1$ in $d_{\perp}=2,3$ and 4 . In this scenario, $\varphi^{2} \approx \tau_{4} \ell_{\|} z^{2} / 2$, and

$$
\eta \simeq-6 \theta^{2} F \beta\left(\frac{z}{\ell_{\perp}}\right)^{2}
$$

This is useful since $F \beta$ is relatively insensitive to $d_{\perp}$. The probability $P(\theta)$ for branes at small angle $\theta$ is related to that for the brane-anti-brane case via

$$
P(\theta) \sim\left(\frac{\sqrt{2}}{\theta}\right)^{d_{\perp}} P(\pi) \sim\left(\frac{4.5 \%}{\theta}\right)^{d_{\perp}}
$$

so $P(\theta)$ is of order unity for $\theta \lesssim 1 / 10$. In Figure 6 , we show the case where $\theta \sim 1 / 6$ and $d_{\perp}=2$. For the diagonal path, the dependence of $n$ of $N_{e}$ is essentially the same as in other scenarios where $\eta \gg \epsilon$,

$$
n \simeq 1-\frac{3}{N_{e}} \quad \frac{d n}{d \ln k} \simeq-\frac{3}{N_{e}^{2}}
$$

Notice that all inflaton paths (see Figure 6) tend to move towards the diagonal path. So we expect only at most a slight correction in $n$ and $\delta_{H}$ for non-diagonal paths.

\subsection{The String Scale}

To see the relation between the string scale $M_{s}$ and $\delta_{H}$, let us consider a particularly interesting scenario. A phenomenologically interesting orientifold construction, either supersymmetric or non-supersymmetric, typically starts with 3 tori. For small $\theta$, we already have 3 sizes : $\ell_{\|}$and $\theta \ell_{\|}$and $\ell_{\perp}$. This fixes the 3 torus sizes. Since the brane resulting from brane collision must wind around $\ell_{\|}$, we may choose, in Eq.(1.1.1.3), $\ell_{\|}=V_{t}$ for 4 -branes, 
or $V_{t}=\theta \ell_{\|}^{2}$ for 5 -branes, where $(2 \pi)^{6} \pi g_{s}^{2} M_{P}^{2}=M_{s}^{2}\left(M_{s} \ell_{\|}\right)^{2}\left(M_{s} \theta \ell_{\|}\right)^{2}\left(M_{s} \ell_{\perp}\right)^{2}$. Assuming that $\ell_{\perp} \gg \ell_{\|}$, we have effective $d_{\perp}=2$. So using Eq.(1.3), Eq.(4.21) for the 5-brane case becomes

$$
\delta_{H}^{2} \simeq \frac{2 g_{s} \theta^{4} F \beta N_{e}^{3}}{75 \pi^{3} \alpha^{3}}\left(\frac{M_{s}}{M_{P}}\right)^{4}
$$

Using $\delta_{H} \sim 1.9 \times 10^{-5}, F \beta \simeq 5$ from Table $1, N_{e} \simeq 60, g_{s} \sim 1, \alpha \simeq 1 / 25$ and $\theta \sim 2 \alpha$, we have

$$
M_{s} \simeq 2 \times 10^{15} \mathrm{GeV}
$$

Here, if $\theta$ is not small, then the probablity to have enough inflation, $P$, will be too small. On the other hand, $\theta$ cannot be much smaller than $2 \alpha$, otherwise $V_{t}$ (and so $g_{s}$ in Eq.(1.3)) will be too big. It is precisely this range of $\theta$ that allows the compactification lattice to play a role, so the overall picture is quite consistent with all data. So, within the crude approximations in a toroidal compactification we have

$$
\delta_{H} \sim 10^{-5} \quad \Longleftrightarrow \quad M_{s} \sim M_{G U T}
$$

This means the coupling unification in MSSM is consistent with brane inflation in the braneworld. A more precise determination of the relation between $\delta_{H}$ and $M_{s}$ will be interesting for phenomenologically realistic compactifications. Note that $\ell_{\perp}>\ell_{\|}$and $M_{s} \theta \ell_{\|} \geq 2 \pi$. If $\ell_{\perp} \gg \ell_{\|}, d_{\perp}$ is effectively 2. Otherwise, we may have non-hypercubic compactification with $d_{\perp}=3$. As we shall see in a moment, the qualitative properties of this case will remain intact. To get a crude estimate of the compactification sizes, let $n_{1}+n_{2}=4+1=5$ for the standard model gauge group. Crudely, with $\theta \simeq u / n_{1}$, we have $M_{s} u r_{\|} \sim 1$ and the 3 compactification radii

$$
u r_{\|}: r_{\|}: r_{\perp} \simeq 1: u^{-1}: 400 g_{s} u
$$

For $\theta \sim 0.1$, we have $u \sim 1 / 3, M_{s} \ell_{\|} \sim 20$ and $M_{s} \ell_{\perp} \sim 10^{3}$. In any case, $M_{s} \lesssim M_{G U T}$ is much closer to the GUT scale than to the electroweak scale. As long as $M_{s}$ is close to $M_{G U T}$, the power-running of the couplings from $r_{\|}^{-1}$ to $M_{s}$ will not mess up the coupling unification [14. In fact, it is even possible that the coupling unification may be improved slightly by a small power-running. A more careful analysis will be interesting.

\subsection{Generic Compactification}

For hypercubic lattice, we see that it is easy to obtain a region that have enough inflation. Since the potential (4.18) has the form $V(\varphi)-A \propto-z^{4}$, it is very flat around the antipodal point $z_{i}=0$, so it is easy to see that a region around $z_{i}=0$ will have enough inflation. For generic compactifications, lower powers of $z_{i}$ appears in the potential. As a result, the potential is much steeper, so naively one may expect the valid region for enough inflation to shrink rapidly to zero around the symmetric point where the net force is zero. However, the actual situation is more robust than naively exected. Here, let us illustrate the situation with a rectangle-like lattice. As shown in Appendix, quadratic terms appear 


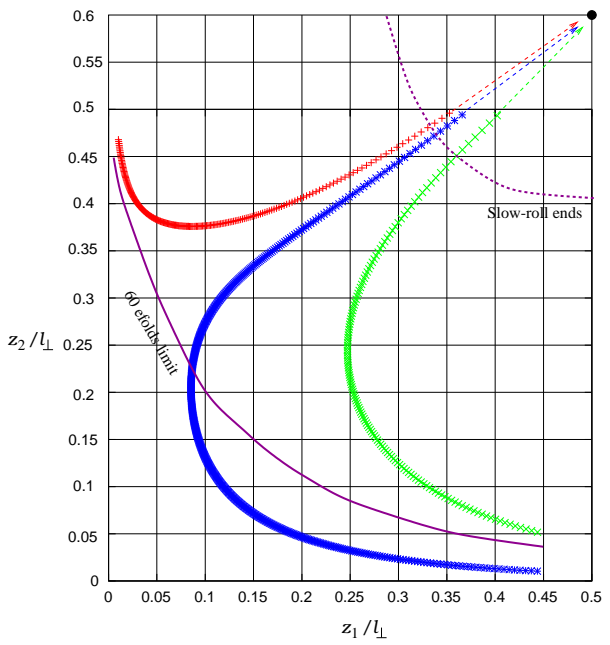

Figure 7: Rectangular lattice with small asymmetry, $a=1.2$. One brane is sitting at the upper right corner. There will be enough inflation if the other brane starts inside the 60 e-fold region. 3 possible trajectories of the inflaton are shown.

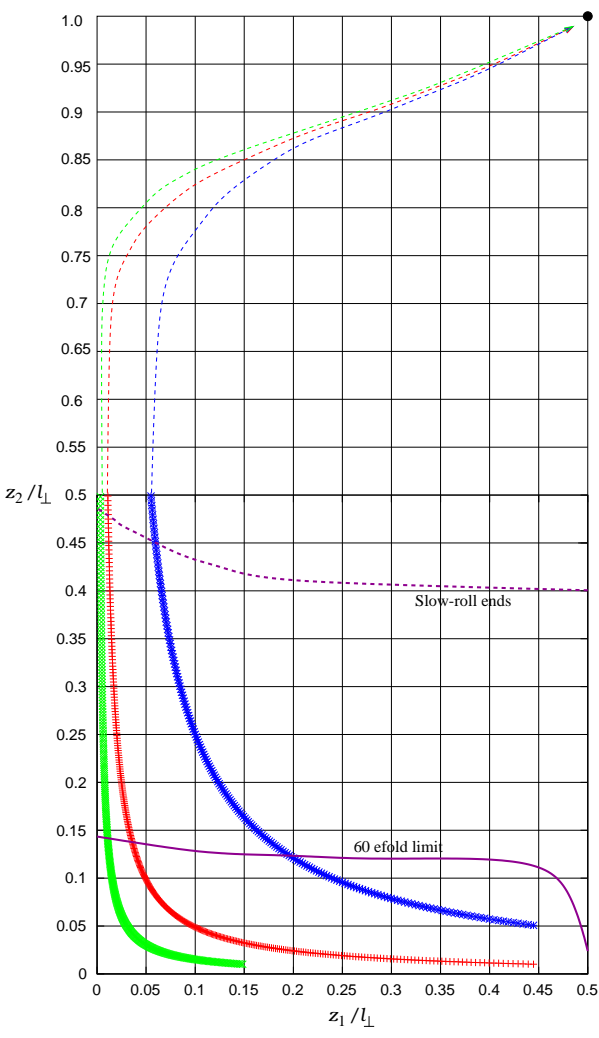

Figure 8: Rectangular lattice with asymmetry $a=2$. 3 possible trajectories of the inflaton are shown. The paths in the slowroll region are reminiscent to renormalization group flows.

in the potential around the symmetric point. Let us consider a 2-dimensional rectangular lattice, with sides $\ell_{\|}$and $a \ell_{\|}$. In this scenario, the effective potential becomes

$$
V(\varphi) \simeq A\left(1-\hat{\lambda}\left[\hat{U}\left(\varphi_{2}^{2}-\varphi_{1}^{2}\right)+D \varphi_{1}^{2} \varphi_{2}^{2}-C\left(\varphi_{1}^{4}+\varphi_{2}^{4}\right)+\cdots\right]\right)
$$

where $U$ is a function of the ratio $a$ of the two sides of the rectangular lattice. Clearly $\hat{U} \rightarrow 0$ as $a \rightarrow 1$. In Table 2 , we show the values $U=\hat{U} /\left(\tau_{p} V_{\|} \ell_{\perp}^{2} / 2\right), C$ and $D$ for $d_{\perp}=2$.

\begin{tabular}{|c|c|c|c|}
\hline $\mathrm{a}$ & $\mathrm{U}$ & $\mathrm{C}$ & $\mathrm{D}$ \\
\hline \hline 1.0 & 0 & 3.94 & 23.64 \\
\hline 1.2 & 0.59 & 2.48 & 14.93 \\
\hline 1.5 & 0.97 & 1.08 & 6.48 \\
\hline 2.0 & 1.03 & 0.23 & 1.40 \\
\hline
\end{tabular}

The inflaton is a two-component field, so the paths for the inflaton can be quite complicated. Typical paths are shown in the figures. In Figure 7 (for $a=1.2$ ) and Figure 8 (for $a=2$ ), one brane is sitting at the upper right corner. If the other brane (at angle 
$\theta \sim 1 / 6$ with respect to the first one) starts inside the 60 e-fold region around the lower left corner, there will be enough inflation. Comparing Figures 7 and 8 to Figure 6, each with the same angle $\theta \sim 1 / 6$, we see that $P$ is mildly sensitive to the shape (complex structure) of the torus

$$
P(\theta, a) \simeq \frac{P(\theta)}{a}
$$

for $a \geq 1$. However, the shape of the 60 e-fold region and the inflaton path do depend strongly on the properties of the torus. In rectangular lattices, the quadratic $z$ (i.e., $\varphi$ ) terms in the potential dominate during the initial part of the inflaton path. If the asymmetry is large, the quadratic term is dominant over a large region, and the inflaton trajectories are simple, as can be seen in Figure 8. When the quartic terms become important they will cause the trajectories to curve towards the corners of the cell. The location of the point where the trajectories turn depends on the asymmetry of the cell. Towards the end of inflation, when the branes are relatively close, the images become negligible and the potential is Coulombic. When the brane separation is comparable to some of the compactified directions, the effects of the Kaluza-Klein modes should be included. It will be interesting to study their effect, although we believe the qualitative features discussed above should remain intact.

By now, it is intuitively clear that the particular hypercubic compactification is not needed for a reasonable value of $P$. Generically, the images of one brane exert forces on the other brane, and there is always a point where the forces balance each other so the inflaton potential is flat at that point. Due to the softness of the brane interaction, there is always a region around that point where the potential is flat enough for sufficient inflation. For initially randomly placed branes at relatively small angle $\theta \lesssim 1 / 10$, which is not hard to arrange for generic compactifications, the probability of sufficient inflation is of order unity. This is very important, since, in any realistic compactification in string model-building, a hypercubic lattice is a very special case.

\subsection{Reheating and Defect Production}

We see that the inflaton and the tachyon modes are charged under the gauge symmetry. In more realistic models, there are chiral fields among the brane modes, which certainly couple to the gauge fields. During reheating after inflation, we expect the inflaton and the tachyon modes to decay to the gauge bosons and the chiral fields. Since the inflaton and the tachyons are brane modes, we expect this reheating process to be very efficient. This picture is essentially that of hybrid inflation.

When $p$-branes collide, tachyons appear and energy is released. Tachyon condensation generically allows the formation of lower-dimensional branes. Following Eq.(2.1,2.2),

$$
V_{T} \propto \operatorname{tr}\left(T T^{\dagger}\right)\left(M_{s}^{4} y^{2}-2 \pi M_{s}^{2} \theta\right)
$$

so when $y$ is small, the open string ground state $T$ becomes tachyonic and the Higgs mechanism (3.10) takes place. Here, the vacuum structure has a $U(N) \times U(N) / U(N)$

symmetry. Generically, lower-dimensional $\mathrm{D}(p-2 k)$-branes can be formed [20] during the brane collision. Since this happens after inflation, it is important to check if the old 
monopole problem returns or not. A naive application of the Kibble mechanism will be disastrous, since that will yield a defect per Hubble volume, resulting in over-abundance of domain walls and monopole-like objects. Fortunately this does not happen, due to the properties of the brane theory.

Imagine a string model that describes our world today. The early universe of this model generically contains branes of all types. The higher-dimensional branes collide to produce lower-dimensional branes and branes that are present today. To be specific, consider a Type IIB orientifold, with D5-branes and orientifold planes. In early universe, there are generically additional D9, D7, D5, D3 and D1-branes with a variety of orientations. (Branes with even $p$ are non-BPS and decay rapidly.) Since the total R-R charges in the compactified directions must be zero, the branes must appear in sets with zero R-R charges, except for D5-branes, which must appear so the total R-R charge between D5branes and orientifold planes is zero. For example, if there are only two 9-branes, they must form a pair of D9-anti-D9-branes. Since D9-branes fill the 9-dimensional space, we expect the D9-anti-D9-brane pair to annihilate first. The resulting tachyon condensation generically produces lower-odd-dimensional branes. Brane on top of each other collides rapidly (unless they are BPS with respect to each other), leaving behind branes separated in the compactified directions.

Consider the last two branes with 3 uncompactified spatial dimensions that are not in today's string ground state. As they approach each other, the universe is in the inflationary epoch. Suppose there are other branes with zero, one or two uncompactified dimensions, which appear as point-like objects, cosmic strings or domain walls, respectively. They are defects in 4-D spacetime. They either annihilate or are inflated away, resulting in negligible densities. After inflation ends, the collision of the two branes with 3 uncompactified spatial dimensions may produce lower-dimensional branes, which appear as defects. A large density of such defects may destroy the nucleosynthesis or even overclose the universe (like the old monopole problem). There are two mechanisms to produce such defects: the Kibble mechanism and thermal production.

After inflation, when the ground state open string mode becomes tachyonic, brane collision and tachyon condensation takes place, and lower-dimensional branes will generically appear via the Kibble mechanism. To be specific, consider a $\mathrm{D} p$-anti- $\mathrm{D} p$-brane collision, which may result in $\mathrm{D}(p-2)$-branes and anti- $\mathrm{D}(p-2)$-branes. At this time, the particle horizon size is typically bigger than the compactification sizes,

$$
\frac{1}{H} \simeq \frac{M_{P}}{M_{s}^{2}} \frac{(2 \pi)^{3 / 2}}{\theta}>>\ell_{\perp}
$$

so the Kibble mechanism does not happen in the compactified directions. In the uncompactified directions, the Kibble mechanism can take place, so cosmic string-like defects may be formed: they are $\mathrm{D}(p-2)$-branes wrapping the same compactified cycles as the original $p$-branes, with one uncompactified dimension. Similarly, if the $\mathrm{D} p$-brane collision can produce $\mathrm{D}(p-4)$-branes, their production will be suppressed since there is less than one Hubble volume in the compactified directions. 
This implies that domain walls and monopole-like objects are not produced by the Kibble mechanism, while cosmic strings may. Generically, there may be closed and stretched cosmic strings, and they form some sort of a network. Fortunately, in contrast to domain walls and monopole-like objects, a cosmic string network may be acceptable [32]. A more careful analysis of the production of cosmic strings will be very interesting. This result is somewhat different from that of Ref. 33], which does not take into account the compactification effect.

Next, let us consider thermal productions. We shall argue that thermal production is negligible. Branes can wrap around the compactification cycles. The mass of such a brane wrapping a p-dimensional volume $V_{p}$, which appears as a point-like object in 4 dimensional spacetime, is

$$
M_{p} \approx \tau_{p} V_{p}=\frac{M_{s} v_{p}}{2 \pi g_{s}} \geq \frac{M_{s}}{g_{s}}
$$

where $v_{p}=M_{s}^{p} V_{p} /(2 \pi)^{p} \geq 1$. Since a wrapped brane is charged, while the total charge of the defects must be zero, they have to be pair-produced or multi-produced. Assuming efficient reheating after inflation, the reheat temperature is given by

$$
T_{R H} \approx\left(\frac{30\left(2 \tau_{p} V_{\|}\right)}{\pi^{2} n_{\text {dof }}}\right)^{1 / 4}
$$

where $n_{d o f}$ is the number of light degrees of freedom at reheating. Typically $n_{d o f}$ is of the order of a few hundred. (For less efficient reheating, the temperature is of course lower.) $T_{R H} \approx M_{s} / 3$ for brane-anti-brane annihilation. Comparing to $M_{p}$, this temperature is probably low enough to prevent the production of defects after inflation. This problem will be totally absent in the collision of branes at angle since the energy released is lower than that from the brane-anti-brane annihilation. Also, if all the compactification directions are larger than the string scale (by a factor of 2 is enough), the defects will be too heavy to be produced. So the defect production is not a problem in this scenario.

\section{Discussions}

Although we find that the string scale is quite close to the GUT scale, this does not imply that a low string scale (say $M_{s}=10 \mathrm{TeV}$ ) is necessarily ruled out. Such a low string scale is possible if the radion grows a lot after inflation 16, 17]. In this scenario, the model necessarily becomes rather complicated.

Keeping the radion mode as a dynamical variable, we can integrate out the $d$ compactified dimensions to obtain the low energy effective 4-dimensional action

$$
S=\int d^{4} x \sqrt{\operatorname{det} g}\left[\frac{e^{d \tilde{\Phi}}}{16 \pi G_{N}}{ }^{(4)} R+\frac{d(d-1)}{16 \pi G_{N}} e^{d \tilde{\Phi}}(\nabla \tilde{\Phi})^{2}-V(\psi, \tilde{\Phi})+\mathcal{L}_{\mathrm{sm}}\left(g_{\mu \nu}, \psi\right)\right]
$$

The action (5.1) has the form of a scalar-tensor theory of gravity, written in the Jordan frame. The Jordan-frame potential $V$ is given by

$$
V(\psi, \tilde{\Phi})=r_{0}^{d} e^{d \tilde{\Phi}} V_{\text {bulk }}(\tilde{\Phi})-\frac{k_{i}}{8 \pi G_{N} r_{0}^{2}} e^{(d-2) \tilde{\Phi}}+V_{\text {brane }}(\psi, \tilde{\Phi}),
$$


where the Ricci scalar of the metric $h_{a b}$ is $2 k_{i} r_{0}^{-2}\left(r_{0}\right.$ being the equilibrium radius of the extra dimensions today) and $k_{i}$ is a dimensionless constant that we may neglect. The action may be written in the Einstein frame,

$S=\int d^{4} x \sqrt{-\hat{g}}\left[\frac{\hat{R}}{16 \pi G}-\frac{1}{2}(\hat{\nabla} \Phi)^{2}-\frac{1}{2} e^{-\Phi / \mu}(\hat{\nabla} \psi)^{2}-e^{-2 \Phi / \mu} V(\psi, \Phi)\right]+S_{\text {rest }}\left[e^{-\Phi / \mu} \hat{g}_{\alpha \beta}, \chi_{\text {rest }}\right]$

where $\hat{g}_{\alpha \beta}$ is the Einstein frame metric, and $g_{\alpha \beta}=e^{-\Phi / \mu} \hat{g}_{\alpha \beta}$ is the physical, Jordan frame metric. Here, $\mu=M_{P} \sqrt{\frac{d+2}{8 d}}$, and the canonically normalized radion field $\Phi$ is related to the radius $r$ of the extra dimensions by $r=r_{0} \exp \left[\frac{\Phi}{d \mu}\right]$. The field $\psi$ is a brane scalar mode. The quantity $V(\psi, \Phi)$ is the Jordan-frame potential for the radion and inflaton (energy per unit proper brane 4 -volume). Finally the action $S_{\text {rest }}\left[g_{\alpha \beta}, \chi_{\text {rest }}\right]$ is the action of the remaining matter fields $\chi_{\text {rest }}$, which may be treated as a fluid.

For generic $V(\Phi)$, the factor $e^{-2 \Phi / \mu}$ will render the potential $e^{-2 \Phi / \mu} V(\Phi)$ unsuitable for inflation. (Such an exponential form is too steep for slow-roll and too shallow for reheating.) However, $\Phi$ may still play a role in an inflationary universe. An example of this scenario has been considered in Ref.[16]. With appropriate $V(\psi, \Phi)$, let $\psi$ be the inflaton, and $\Phi$ be frozen during the inflationary epoch. After inflation, $\Phi$ grows, resulting in today's value for the Newton's constant, which is substantially smaller than the effective Newton's constant during inflation. This allows the correct value for density perturbation even though the string scale can be much smaller than what we find earlier. Of course, this scenario is much more complicated and model-dependent than that discussed in this paper.

\section{Summary and Remarks}

In brane inflation, the inter-brane separations play the role of inflatons, while the bulk modes provide the brane interactions that generate the inflaton potential. Such properties are well studied in string theory. By itself, the resulting potential is generically unsuitable for inflation. Fortunately, any realistic phenomenological string realization requires the compactification of the extra dimensions. Also, the weak coupling behavior observed in nature probably requires the branes to have extra dimensions (beyond the 3 uncompactified spatial dimensions) that wrap around some of the compactification directions. These two properties dramatically improves the brane inflationary scenario. Without any fine-tuning, the probability of randomly placed branes in the early universe to originate substantial inflation can easily be of order unity. This allows one to argue that brane interaction provides an explanation of the origin of inflation.

In a simple brane inflationary scenario suggested by our analysis, we find that the amplitude of the density perturbation observed by COBE implies that the string scale is very close to the GUT scale. A more careful analysis in more realistic string models (e.g., orientifolds) can make this relation more precise. We can reverse the analysis: starting with MSSM and coupling unification, with $M_{s}=M_{G U T}$, we find that a generic orientifold model will yield a density perturbation in CMB of the correct magnitude. 
A number of other issues also deserve further analysis, e.g., the likely braneworld just before inflation, the reheating and the defect production after inflation, $p$-brane- $p$-brane interactions, the generation of density perturbation due to multi-component feature of the inflaton, as is likely to be the case in the braneworld. We hope that this work shows that further studies along this direction is worthwhile.

We thank Keith Dienes, Sash Sarangi, Ashoke Sen, Gary Shiu and Ira Wasserman for discussions. This research was partially supported by the National Science Foundation.

\section{A. Summing Over The Lattice}

Here we provide some details to the derivation of the potential via summing over the lattice. The potential for the D-dimensional lattice is given by:

$$
V(\vec{r})=\left\{\begin{array}{l}
A-B \sum_{i} \frac{1}{\left|\vec{r}-\vec{r}_{i}\right|^{d_{\perp}-2}}, d_{\perp}>2 \\
A+B \sum_{i} \ln \left|\vec{r}-\vec{r}_{i}\right|, d_{\perp}=2
\end{array}\right.
$$

where the sum is over all the lattice sites. In order to estimate the potential around the antipodal point we expand to 4 th order in $\vec{z}$, around the center of the elementary cell.

$$
\sum_{i} \frac{1}{\left|\vec{z}-\vec{r}_{i}\right|^{d_{\perp}}}=\sum_{i} \frac{1}{r_{i}^{d_{\perp}}\left[1-2 \vec{z} \cdot \vec{r}_{i} / r_{i}^{2}+z^{2} / r_{i}^{2}\right]^{d_{\perp} / 2}}
$$

denoting $\delta=-2 \vec{z} \cdot \vec{r}_{i} / r_{i}^{2}+z^{2} / r_{i}^{2}$ and $p=d_{\perp}-2>0$ we have:

$$
\begin{aligned}
& \sum_{i} \frac{1}{\left|\vec{z}-\vec{r}_{i}\right|^{p}}=\sum_{i}\left[\frac{1}{\left(r_{i}^{2}\right)^{p / 2}}-\frac{p}{2} \frac{\delta}{\left(r_{i}^{2}\right)^{p / 2+1}}+\frac{p}{2}\left(\frac{p}{2}+1\right) \frac{1}{2 !} \frac{\delta^{2}}{\left(r_{i}^{2}\right)^{p / 2+2}}\right. \\
& -\frac{p}{2}\left(\frac{p}{2}+1\right)\left(\frac{p}{2}+2\right) \frac{1}{3 !} \frac{\delta^{3}}{\left(r_{i}^{2}\right)^{p / 2+3}} \\
& \left.+\frac{p}{2}\left(\frac{p}{2}+1\right)\left(\frac{p}{2}+2\right)\left(\frac{p}{2}+3\right) \frac{1}{4 !} \frac{\delta^{4}}{\left(r_{i}^{2}\right)^{p / 2+4}}+\cdots\right]
\end{aligned}
$$

For $p=0\left(d_{\perp}=2\right)$, the factor of $p$ in every term should be dropped. Using the expression for $\delta$ and grouping the 4 th powers of $z$ we obtain:

$$
\begin{aligned}
& V_{4}(\vec{z})=\frac{1}{4 !}\left[-p(p+2) \sum_{i} \frac{3 z^{4}}{r_{i}^{p+4}}+p(p+2)(p+4) \sum_{i} \frac{6 z^{2}\left(\vec{z} \cdot \vec{r}_{i}\right)^{2}}{r_{i}^{p+6}}\right. \\
& \left.-p(p+2)(p+4)(p+6) \sum_{i} \frac{\left(\vec{z} \cdot \vec{r}_{i}\right)^{4}}{r_{i}^{p+8}}\right]
\end{aligned}
$$

As an example, for a two-dimensional square lattice, we express $\vec{z}$ in terms of the components, $\vec{z}=\left(z_{1}, z_{2}\right)$ and obtain:

$$
V_{4}\left(z_{1}, z_{2}\right)=\frac{1}{4 !}\left(z_{1}^{4}+z_{2}^{4}\right)\left[\sum_{i, j} \frac{48\left(i+\frac{1}{2}\right)^{2}\left(j+\frac{1}{2}\right)^{2}}{\left(\left(i+\frac{1}{2}\right)^{2}+\left(j+\frac{1}{2}\right)^{2}\right)^{4}}-\sum_{i, j} \frac{6}{\left(\left(i+\frac{1}{2}\right)^{2}+\left(j+\frac{1}{2}\right)^{2}\right)^{2}}\right]
$$




$$
-\frac{1}{4 !} z_{1}^{2} z_{2}^{2}\left[\sum_{i, j} \frac{288\left(i+\frac{1}{2}\right)^{2}\left(j+\frac{1}{2}\right)^{2}}{\left(\left(i+\frac{1}{2}\right)^{2}+\left(j+\frac{1}{2}\right)^{2}\right)^{4}}-\sum_{i, j} \frac{36}{\left(\left(i+\frac{1}{2}\right)^{2}+\left(j+\frac{1}{2}\right)^{2}\right)^{2}}\right]
$$

Similarly, in the case of a four dimensional hypercubic lattice, $\vec{z}=\left(z_{1}, z_{2}, z_{3}, z_{4}\right)$, and the potential is:

$$
\begin{aligned}
& V_{4}\left(z_{1}, z_{2}, z_{3}, z_{4}\right)=\frac{1}{4 !}\left(z_{1}^{4}+\cdots+z_{4}^{4}\right)\left[192 \sum_{i, j, k, l} \frac{\left(i+\frac{1}{2}\right)^{2}\left(j+\frac{1}{2}\right)^{2}+\cdots+\left(k+\frac{1}{2}\right)^{2}\left(l+\frac{1}{2}\right)^{2}}{\left(\left(i+\frac{1}{2}\right)^{2}+\cdots+\left(l+\frac{1}{2}\right)^{2}\right)^{5}}\right. \\
& \left.-\sum_{i, j, k, l} \frac{48}{\left(\left(i+\frac{1}{2}\right)^{2}+\cdots+\left(l+\frac{1}{2}\right)^{2}\right)^{3}}\right] \\
& -\frac{1}{4 !}\left(z_{1}^{2} z_{2}^{2}+\cdots+z_{3}^{2} z_{4}^{2}\right)\left[384 \sum_{i, j, k, l} \frac{\left(i+\frac{1}{2}\right)^{2}\left(j+\frac{1}{2}\right)^{2}+\cdots+\left(k+\frac{1}{2}\right)^{2}\left(l+\frac{1}{2}\right)^{2}}{\left(\left(i+\frac{1}{2}\right)^{2}+\cdots+\left(l+\frac{1}{2}\right)^{2}\right)^{5}}\right. \\
& \left.-\sum_{i, j, k, l} \frac{96}{\left(\left(i+\frac{1}{2}\right)^{2}+\cdots+\left(l+\frac{1}{2}\right)^{2}\right)^{3}}\right]
\end{aligned}
$$

If the lattice is not hypercubic, the leading term in the expansion is the 2nd order term. Using a two-dimensional rectangular lattice as example, we write the vectors of the lattice as $\vec{r}_{i, j}=(i+1 / 2) \hat{z_{1}}+a(j+1 / 2) \hat{z_{2}}$, where $\hat{z_{1}}$ and $\hat{z_{2}}$ are the unit vectors in the $z_{1}$ and $z_{2}$ directions and $a$ is the ratio of the two sides of the rectangular lattice. The second-order term of the expansion of the potential is:

$$
V_{2}\left(z_{1}, z_{2}\right)=\left(z_{1}^{2}-z_{2}^{2}\right) \frac{1}{2 !} \sum_{i, j} \frac{a^{2}\left(j+\frac{1}{2}\right)^{2}-\left(i+\frac{1}{2}\right)^{2}}{\left(i+\frac{1}{2}\right)^{2}+a^{2}\left(j+\frac{1}{2}\right)^{2}}
$$

We see that $V_{2}\left(z_{1}, z_{2}\right)$ becomes zero if $a=1$.

We note that summing over the lattice will generically induce a correction to the constant $A$ in the potential. However, this correction is typically quite small and will be ignored.

\section{References}

[1] A. H. Guth, Phys. Rev. D23 (1981) 347;

A. D. Linde, Phys. Lett. B108 (1982) 389;

A. Albrecht and P. J. Steinhardt, Phys. Rev. Lett. 48 (1982) 1220.

[2] G.F. Smoot et. al., Astrophy. J. 396 (1992) L1;

C.L. Bennett et. al., 4-year COBE DMR Cosmic Microwave Background Observations: Maps and Basic Results, Astrophy. J. 464 (1996) L1, astro-ph/9601067.

[3] A.T. Lee et. al. (MAXIMA-1), A High Spatial Resolution Analysis of the MAXIMA-1 Cosmic Microwave Background Anisotropy Data, astro-ph/0104459. 
C.B. Netterfield et. al. (BOOMERANG), A measurement by BOOMERANG of multiple peaks in the angular power spectrum of the cosmic microwave background, astro-ph/0104460; C. Pryke, et. al. (DASI), Cosmological Parameter Extraction from the First Season of Observations with DASI, astro-ph/0104490.

[4] See e.g., A.R. Liddle and D.H. Lyth, Cosmological Inflation and Large-Scale Structure, Cambridge University Press, 2000.

[5] G. Dvali and S.-H.H. Tye, Brane inflation, Phys. Lett. B450 (1999) 72, hep-ph/9812483.

[6] J. Polchinski, String Theory, Vols 1 \& 2, Cambridge University Press, 1998.

[7] G. Dvali, Q. Shafi and S.Solganik, D-Brane Inflation, hep-th/0105203.

[8] C. P. Burgess, M. Majumdar, D. Nolte, F. Quevedo, G. Rajesh and R. J. Zhang, The Inflationary Brane-Antibrane Universe, JHEP 07 (2001) 047, hep-th/0105204.

[9] J. Garcia-Bellido, R. Rabadan, F. Zamora, Inflationary Scenarios from Branes at Angles, JHEP 01 (2002) 036, hep-th/0112147.

[10] S. H. Alexander, Inflation from D- $\bar{D}$ Brane Annihilation, Phys. Rev. D65 (2002) 023507, hep-th/0105032;

E. Halyo, Inflation from rotation, hep-ph/0105341,

G. Shiu and S.-H. H. Tye, Some Aspects of Brane Inflation, Phys. Lett. B516 (2001) 421, hep-th/0106274;

A. Mazumdar, S. Panda, A. Perez-Lorenzana, Assisted inflation via tachyon condensation, Nucl. Phys. B614 (2001) 101, hep-ph/0107058;

C. Herdeiro, S. Hirano and R. Kallosh, String Theory and Hybrid Inflation/Acceleration, JHEP 0112 (2001) 027, hep-th/0110271;

B.-S. Kyae and Q. Shafi, Branes and Inflationary Cosmology, Phys. Lett. B526 (2002) 379, hep-ph/0111101;

C. P. Burgess, P. Martineau, F. Quevedo, G. Rajesh and R.-J. Zhang, Brane-Antibrane Inflation in Orbifold and Orientifold Models, hep-th/0111025;

R. Blumenhagen, B. Kors, D. Lust and T. Ott, Hybrid Inflation in Intersecting Brane Worlds, hep-th/0202124;

K. Dasgupta, C. Herdeiro, S. Hirano and R. Kallosh, D3/D7 Inflationary Model and M-theory, hep-th/0203019.

[11] S. Dimopoulos, S. Raby and F. Wilczek, Phys. Rev. D24 (1981) 1681;

U. Amaldi, W. de Boer and H. Furstenau, Phys. Lett. B260 (1991) 447;

C. Giunti, C.W. Kim and U.W. Lee, Mod. Phys. Lett. A6 (1991) 1745;

P. Langacker and M. Luo, Phys. Rev. D44 (1991) 817;

J. Ellis, S. Kelley and D.V. Nanopoulos, Phys Lett. B249 (1990) 441.

[12] G. Shiu and S.-H. H. Tye, TeV Scale Superstring and Extra Dimensions, Phys. Rev. D58 (1998) 106007, hep-th/9805157.

[13] S.P. de Alwis, Coupling of branes and normalization of effective actions in string/M-theory, Phys. Rev. D56 (1997) 7963, hep-th/9705139;

E. Caceres, V.S. Kaplunovsky and I.M. Mandelberg, Large-Volume String Compactifications, Revisited, Nucl. Phys. B493 (1997) 73, hep-th/9606036;

N. Sakai and M. Abe, Prog. Theor. Phys. 80 (1988) 162. 
[14] K.R. Dienes, E. Dudas and T. Gherghetta, Extra Spacetime Dimensions and Unification, Phys. Lett. B436 (1998) 55, hep-ph/9803466;

Z. Kakushadze and T.R. Taylor, Higher Loop Effects on Unification via Kaluza-Klein Thresholds, Nucl.Phys. B562 (1999) 78, hep-th/9905137.

[15] N. Arkani-Hamed, S. Dimopoulos and G. Dvali, The hierarchy problem and new dimensions at a millimeter, Phys. Lett. B429 (1998) 263, hep-ph/9803315;

I. Antoniadis, N. Arkani-Hamed, S. Dimopoulos and G. Dvali, New dimensions at a millimeter to a Fermi and superstrings at a TeV, Phys. Lett. B436 (1998) 257, hep-ph/9804398.

[16] E.E. Flanagan, S.-H. H. Tye and I. Wasserman, A Cosmology of the Brane World, Phys. Rev. D62 (2000) 024011, hep-ph/9909373.

[17] G. Dvali, Infrared Hierarchy, Thermal Brane Inflation and Superstrings as Superheavy Dark Matter, Phys. Lett. B459 (1999) 489, hep-ph/9905204;

A. Mazumdar, Extra Dimensions and Inflation, Phys. Lett. B469 (1999) 55, hep-ph/9902381;

N. Arkani-Hamed, S. Dimopoulos, N. Kaloper and J. March-Russell, Rapid Asymmetric Inflation and Early Cosmology in Theories with Sub-Millimeter Dimensions, Nucl. Phys. B567 (2000) 189, hep-ph/9903224.

[18] A. Linde, Hybrid Inflation, Phys. Rev. D49 (1994) 748, astro-ph/9307002.

[19] A. Sen, Stable non-BPS bound states of BPS D-branes, JHEP 9808 (1998) 010, hep-th/9805019; $S O(32)$ spinors of type I and other solitons on brane-antibrane pair, JHEP 9809 (1998) 023, hep-th/9808141.

[20] E. Witten, D-branes and K-theory, JHEP 9812 (1998) 019, hep-th/9810188; P. Horava, Type IIA D-branes, K-theory, and matrix theory, Adv. Theor. Math. Phys. 2 (1999) 1373, hep-th/9812135.

[21] R. C. Myers, Dielectric-branes, JHEP 9912 (1999) 022, hep-th/9910053.

[22] T. Banks and L. Susskind, Brane-Antibrane Forces, hep-th/9511194.

[23] N. Berkovits, The tachyon potential in open Neveu-Schwarz string field theory, JHEP 0004 (2000) 022, hep-th/0001084.

[24] N. Berkovits, A. Sen and B. Zwiebach, Tachyon condensation in superstring field theory, Nucl. Phys. B587 (2000) 147, hep-th/0002211.

[25] D. Kutasov, M. Marino and G. W. Moore, Some exact results on tachyon condensation in string field theory, JHEP 0010 (2000) 045, hep-th/0009148; Remarks on tachyon condensation in superstring field theory, hep-th/0010108;

P. Kraus and F. Larsen, Boundary string field theory of the DD-bar system, Phys. Rev. D63 (2001) 106004, hep-th/0012198;

T. Takayanagi, S. Terashima and T. Uesugi, Brane-antibrane action from boundary string field theory, JHEP 0103 (2001) 019, hep-th/0012210.

[26] A. Sen, Tachyon condensation on the brane antibrane system, JHEP 9808 (1998) 012, hep-th/9805170.

[27] J. Polchinski, Dirichlet-Branes and Ramond-Ramond Charges, Phys. Rev. Lett. 75 (1995) 4724, hep-th/9510017. 
[28] M. Berkooz, M.R. Douglas and R.G. Leigh, Branes Intersecting at Angles, Nucl. Phys. B480 (1996) 265, hep-th/9606139;

H. Arfaei and M.M.S. Jabbari, Different D-brane Interactions, Phys. Lett B 394 (1997) 288, hep-th/9608167.

[29] Z. Kakushadze and S.-H.H. Tye, Brane World, Nucl. Phys. B548 (1999) 180, hep-th/9809147.

[30] L. Randall and R. Sundrum, A large mass hierarchy from a small extra dimension, Phys. Rev. Lett. 83 (1999) 3370, hep-ph/9905221;

An alternative to compactification, Phys. Rev. Lett. 83 (1999) 4690, hep-th/9906064.

[31] See e.g., R. Blumenhagen, L. Goerlich, B. Koers and D. Luest, Noncommutative Compactifications of Type I String on Tori and Magnetic Background Flux, JHEP 0010 (2000) 006, hep-th/0007024;

S. Förste, G. Honecker and R. Schreyer, Supersymmetric $Z_{N} \times Z_{M}$ Orientifolds in 4-D with D-branes at Angles, Nucl. Phys. B593 (2001) 127, hep-th/0008250;

G. Aldazabal, S. Franco, L. E. Ibanez, R. Rabadan, A. M. Uranga, Intersecting Brane Worlds, JHEP 0102 (2001) 047, hep-ph/0011132;

R. Blumenhagen, B. Koers and D. Luest, Type I Strings with F and B Flux, JHEP 0102 (2001) 030, hep-th/0012156;

R. Blumenhagen, B. Körs, D. Lüst and T. Ott, The Standard Model from Stable Intersecting Brane World Orbifolds, Nucl. Phys. B616 (2001) 3, hep-th/0107138;

M. Cvetic, G. Shiu and A. M. Uranga, Three-Family Supersymmetric Standard-like Models from Intersecting Brane Worlds Phys. Rev. Lett. 87 (2001) 201801, hep-th/0107143.

[32] See e.g., E.W. Kolb and M.S. Turner, The Early Universe, Addison-Wesley Publ. Co., 1990.

[33] M. Majumdar and A. Christine-Davis, Cosmological Creation of D-branes and anti-D-branes, hep-th/0202148. 